\title{
Differences between Mitiglinide/Voglibose Fixed- dose Combination and Glimepiride in Modifying Low- density Lipoprotein Heterogeneity in Japanese Type-2 Diabetic Patients: A Pilot Study
}

Authors

Affiliations

\author{
S. Tani ${ }^{1,2}$, K. Nagao ${ }^{2}$, A. Hirayama
}

${ }^{1}$ Department of Health Planning Center, Nihon University Hospital, Tokyo Japan

2Division of Cardiology, Department of Medicine, Nihon University School of Medicine, Tokyo Japan
Key words

glimepiride

- LDL-heterogeneity

- mitiglinide/voglibose fixed-dose combination

received 24.03.2015

accepted 26.04.2015

\section{Bibliography}

DOI http://dx.doi.org/

10.1055/s-0035-1549993

Published online:

May 26, 2015

Drug Res 2016;

66: 94-99

(c) Georg Thieme Verlag KG

Stuttgart · New York

ISSN 2194-9379

\section{Correspondence}

\section{S. Tani, MD}

Department of Health Planning

Center

Nihon University Hospital

1-6 Kanda-Surugadai

Chiyoda-ku

Tokyo 101-8309

Japan

Tel.: + 81/3/3293 1701

Fax: $+81 / 3 / 32931708$

tani.shigemasa@nihon-u.ac.jp

\section{Abstract}

$\nabla$

Purpose: The purpose of this study was to compare the effects of mitiglinide/voglibose fixed-dose combination and glimepiride on low-density lipoprotein (LDL)-heterogeneity in type-2 diabetic patients with unstable glycemic control after treatment with dipeptidyl peptidase-4 (DPP-4) inhibitors.

Methods: This was an open-label pilot study in which type- 2 diabetic patients were randomly assigned to the mitiglinide/voglibose (fixed-dose combination of mitiglinide $10 \mathrm{mg}$ and voglibose $0.2 \mathrm{mg}, \mathrm{n}=14)$ or glimepiride $(0.5 \mathrm{mg}, \mathrm{n}=16)$.

Results: In the glimepiride group, serum LDL cholesterol (LDL-C) and small-dense (sd) LDL levels decreased significantly $(-8.5 \%$ and $-9.0 \%)$, while sd-LDL/LDL-C and an indicator of LDLparticle size, LDL-C/apoB, did not change sig-

\section{Introduction}

$\nabla$

In recent years, it has become recognized that it is important to control hemoglobin $\mathrm{A} 1 \mathrm{c}$ (HbA1c) levels below $7.0 \%$ for preventing diabetes-associated complications based on evidence accumulated in several clinical studies [1,2], and the appearance of dipeptidyl peptidase-4 (DPP-4) inhibitors made it easier than before to control blood glucose levels. However, there is a rebound phenomenon of the effect of DPP-4 inhibitors to reduce blood glucose levels or non-responders to DPP-4 inhibitors, and further glycemic control is required in not a few patients [3].

It is known that arteriosclerosis progresses with increasing speed in diabetic patients due to the high proportion of the small-particle LDL which strongly induces arteriosclerosis, and that the prognosis of arteriosclerotic cardiovascular disease, particularly coronary artery disease, complicated by diabetes is poor $[4,5]$. nificantly. In the mitiglinide/voglibose group, serum LDL-C levels did not change, while sd-LDL levels and sd-LDL/LDL-C decreased significantly $(-8.6 \%$ and $-7.9 \%)$ and LDL-C/apoB increased significantly (5.8\%). Fasting blood glucose levels tended to be reduced to a greater extent in the glimepiride group than in the mitiglinide/voglibose group $(-13.9 \%$ vs. $-8.4 \%, p=0.08)$, while the rate of reduction of $\mathrm{HbA} 1 \mathrm{c}$ levels tended to be higher in the mitiglinide/voglibose group than in the glimepiride group ( $-6.9 \%$ vs. $-3.4 \%$, $\mathrm{p}=0.09$ ), suggesting differences in fluctuating blood glucose levels between the 2 groups.

Conclusion: There were differences in the effects of mitiglinide/voglibose fixed-dose combination and glimepiride in addition to DPP-4 inhibitors on LDL-metabolism, and this may be related to fluctuations in blood glucose levels after treatment with these agents.

Glimepiride is a long-acting agent widely used in combination with DPP-4 inhibitors which mainly robustly reduces fasting blood glucose (FBG) levels [6], and its effect on lipid profiles has been investigated [7-10], On the other hand, mitiglinide/voglibose fixed-dose combination is a drug combining a rapid- and short-acting insulin secretagogue (glinide) and a postprandial hyperglycemia-improving agent ( $\alpha$-glucosidase inhibitor) which strongly suppresses the postprandial elevation of blood glucose levels: its effect on lipid profiles, particularly on LDL-heterogeneity, has not been investigated. In addition, mitiglinide/voglibose fixed-dose combination reduces the area under the blood glucose response curve (glucose AUC) through suppression of the postprandial elevation of blood glucose levels [11]. It was also reported that a reduction in blood glucose AUC reduces serum sd-LDL levels [12]. We hypothesized that mitiglinide/voglibose fixed-dose combination, which suppresses the 
postprandial elevation of blood glucose levels and thereby reduces the daily fluctuation range of blood glucose levels in type-2 diabetic patients, may improve LDL-heterogeneity. The purpose of this study was to compare the effects of additional administration of mitiglinide/voglibose fixed-dose combination and glimepiride on changes in LDL-heterogeneity in type-2 diabetic patients with unstable glycemic control after treatment with DPP-4 inhibitors.

\section{Methods \\ $\nabla$ \\ Patients}

The study period was from April 1 to October 30, 2013. The subjects were patients who had been diagnosed as having type- 2 diabetes, who had or had not received antidiabetic drugs, and who were receiving DPP-4 inhibitors due to poor glycemic control although diet and exercise therapy was recommended, and in whom HbA1c levels increased or did not decrease to $<7.0 \%$ after treatment with DPP-4 inhibitors.

\section{Study design}

This was a single-center, randomized, open-label pilot study in which type 2 diabetic patients receiving DPP-4 inhibitors were randomly assigned to the mitiglinide/voglibose group (a fixeddose combination of mitiglinide $10 \mathrm{mg}$ and voglibose $0.2 \mathrm{mg}$; $\mathrm{n}=17)$ or glimepiride $(0.5 \mathrm{mg} / \mathrm{day} ; \mathrm{n}=18)$ group. The envelope method was used for randomization. Primary endpoints were changes in serum sd-LDL levels and the sd-LDL proportion (the ratio of sd-LDL to LDL-C), which are indicators of LDL-heterogeneity, and changes in LDL-C/apoB, which is a rough marker of LDL particle size [13]. Secondary endpoints were changes in FBG and HbA1c levels. Patients who had already received glimepiride, $\alpha$-glucosidase inhibitors, or glinide, those with malignant neoplasms, and those who were considered unsuitable to participate in this study were excluded. There were no changes or additions to any of the anti-diabetic drugs, or lipid-modifying drugs during the period of this trial. Blood and urine tests were performed at the start of drug therapy and 12-weeks after the start. Compliance was verbally confirmed in terms of how often the patient took the study drug.
The study protocol was approved by the Institutional Review Boards at our institute and written informed consent was obtained from all participants.

\section{Laboratory measurements}

Fasting blood samples were collected early in the morning after a 12-h fast. The serum sd-LDL level was measured by an enzymatic homogeneous assay with an automated analyzer (SRL Co., Ltd., Tokyo, Japan). The serum total cholesterol (TC), high-density lipoprotein cholesterol (HDL-C), and triglyceride (TG) levels were measured by the standard methods. The serum LDL-C level was estimated by using the Friedewald formula [14]. The serum apolipoprotein (apo) level was determined by turbidimetric latex agglutination assays (Daiichi Pure Chemicals Co., Ltd., Tokyo, Japan).

\section{Statistical analysis}

We performed all of the statistical analyses by using the SPSS Window ver 12.0 software program (Statistical Package for the Social Sciences, SPSS Inc., Chicago, IL). Data were expressed as the mean \pm standard deviation for continuous variables and as percentages for discrete variables. Differences between the groups in data for continuous variables were analyzed by Student's $t$-test, and the data for categorical variables were analyzed by the $\mathrm{X}^{2}$ test. $\mathrm{P}$ values less than 0.05 were considered to indicate statistical significance.

\section{Results \\ $\nabla$}

\section{Patient characteristics}

The mean interval between the administration of DPP- 4 inhibitors and randomization was $8.3 \pm 2.5$ months. One patient was withdrawn because this patient refused mitiglinide/voglibose in the mitiglinide/voglibose group. In the glimepiride group, pertinent baseline data were not available for 1 patient. After exclusion of these patients, the data of 30 patients each in the 2 groups were subjected to this analysis, and $\odot$ Table 1 summarizes their baseline characteristics. There were no significant differences between the 2 study groups.

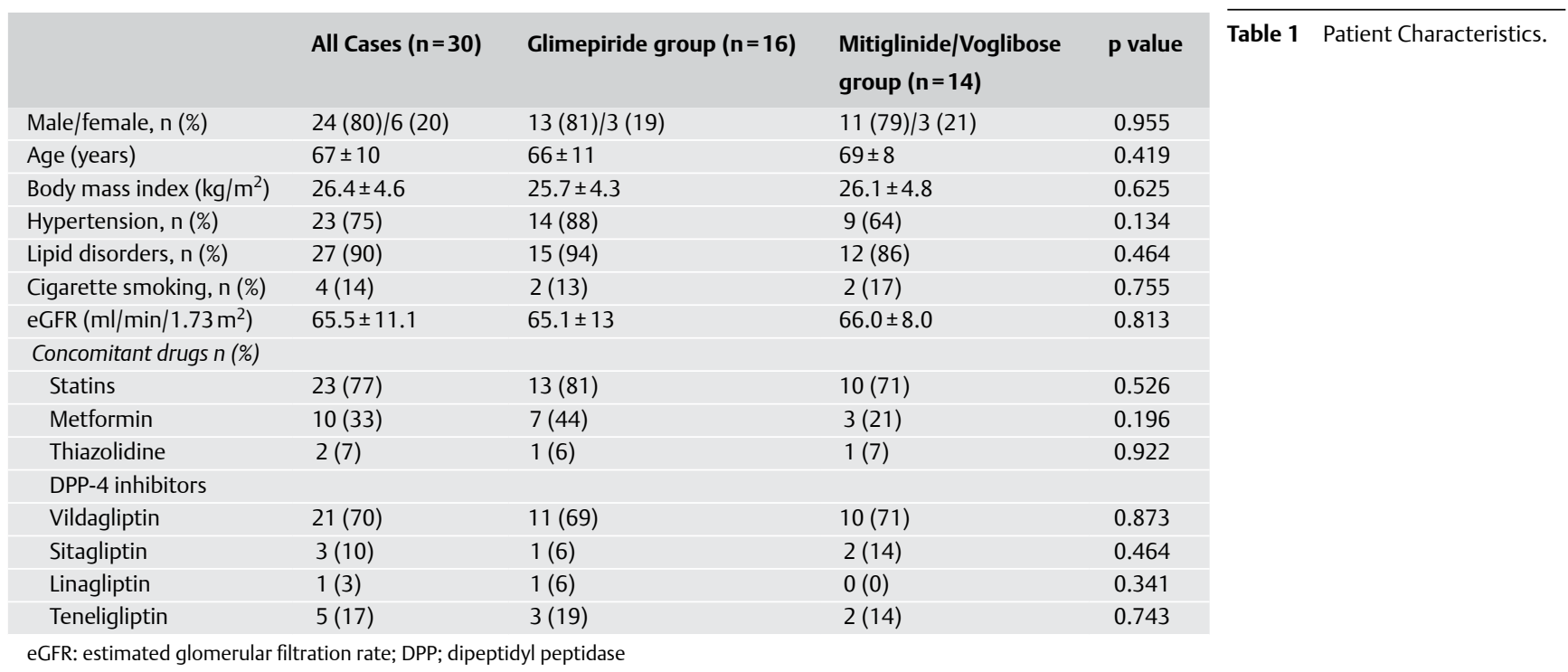






All of the patients included in this study were followed up at 2-month intervals, and were confirmed to show no noticeable changes of FBG or HbA1c levels. Therefore, we consider that there was no problem in respect of the subjects' adherence to the mtiglinide/voglibose fixed-dose combination treatment.

\section{Changes in sd-LDL and sd-LDL proportion}

In the mitiglinide/voglibose group, there were no significant changes in serum LDL-C levels ( $\mathrm{p}=0.587$ ), whereas serum sd LDL levels $(p=0.045)$ and the sd-LDL proportion $(p=0.01)$ decreased significantly. In the glimepiride group, serum LDL-C levels decreased significantly $(p=0.003)$ with a significant decrease in serum sd LDL levels $(\mathrm{p}=0.027)$, but there were no significant changes in the sd-LDL proportion $(p=0.562)$. On the other hand, apoB levels decreased significantly in both groups $(\mathrm{p}=0.040$ and 0.006) ( $\odot$ Fig. 1 and $\odot$ Table 2).

\section{Changes in estimated LDL particle size}

The LDL-C/apoB ratio, which is an indicator of LDL particle size, increased significantly in the mitiglinide/voglibose group $(p=0.002)$, but did not change significantly in the glimepiride group $(\mathrm{p}=0.560)$. In addition, in patients with an LDL-C/apoB ratio of $\geq 1.2$, the ratio increased significantly 12 weeks after treatment compared with baseline in the mitiglinide/voglibose group ( $p=0.025$ ), but did not increase significantly in the glimepiride group ( $\mathrm{p}=0.070$ ) ( $\odot$ Fig. 2 and $\odot$ Table 2 ).

\section{Changes in glycemic control}

FBG and HbA1c levels decreased significantly and the mean HbA1c level could be maintained below $7.0 \%$ in both groups. There were no statistically significant differences in the rate of change in fasting blood glucose or HbA1c levels between the 2 groups. However, the rate of reduction of FBG levels tended to be higher in the glimepiride group than in the mitiglinide/voglibose group $(p=0.08)$, whereas the rate of reduction of HbA1c levels tended to be higher in the mitiglinide/voglibose group than in the glimepiride group $(\mathrm{p}=0.09)$ ( $\diamond$ Table 2 ).

\section{Safety}

There were no adverse events of any severity, or abnormal changes in laboratory measurements with or without possible causal relationship to the study medications during the study period.

\section{Discussion}

In this study, we revealed the following: additional administration of mitiglinide/voglibose fixed-dose combination or glimepiride reduced serum sd-LDL levels in patients with unstable glycemic control after treatment with DPP-4 inhibitors in patients with type-2 diabetes. However, mitiglinide/voglibose fixed-dose combination significantly reduced the sd-LDL proportion and increased an indicator of LDL-particle size, LDL-C/ apoB, while glimepiride did not change the sd-LDL proportion or LDL-C/apoB significantly, suggesting that these agents affect LDL-metabolism differently. Moreover, mitiglinide/voglibose fixed-dose combination, like glimepiride, was suggested to be useful for glycemic control as an agent used in combination with DPP-4 inhibitors.

It has been reported that glimepiride reduces serum LDL-C levels [6-10] and that this effect is exerted by enhancing LDL hyper- 
Table 2 Changes in Laboratory Profiles.

\begin{tabular}{|c|c|c|c|c|}
\hline Variables & Baseline & 12 Week & $\begin{array}{l}\text { Change from } \\
\text { baseline (\%) }\end{array}$ & p Value \\
\hline \multicolumn{5}{|l|}{ FBG (mg/dL) } \\
\hline Glimepiride & $143 \pm 18$ & $124 \pm 22$ & $-13.9 \pm 8.8$ & $<0.0001$ \\
\hline \multirow[t]{2}{*}{ Mitiglinide/Voglibose } & $141 \pm 36$ & $128 \pm 29$ & $-8.2 \pm 7.0$ & 0.001 \\
\hline & 0.851 & 0.621 & 0.059 & \\
\hline \multicolumn{5}{|l|}{ HbA1c (\%) } \\
\hline Glimepiride & $7.11 \pm 0.68$ & $6.86 \pm 0.62$ & $-3.4 \pm 5.3$ & 0.024 \\
\hline \multirow[t]{2}{*}{ Mitiglinide/Voglibose } & $7.03 \pm 0.77$ & $6.51 \pm 0.46$ & $-6.9 \pm 5.8$ & 0.002 \\
\hline & 0.772 & 0.101 & 0.094 & \\
\hline \multicolumn{5}{|l|}{$\mathrm{TC}(\mathrm{mg} / \mathrm{dL})$} \\
\hline Glimepiride & $184 \pm 32$ & $176 \pm 25$ & $-3.7 \pm 8.4$ & 0.069 \\
\hline \multirow[t]{2}{*}{ Mitiglinide/Voglibose } & $168 \pm 25$ & $168 \pm 21$ & $0.5 \pm 10.8$ & 0.929 \\
\hline & 0.139 & 0.341 & 0.240 & \\
\hline \multicolumn{5}{|l|}{ LDL-C (mg/dL) } \\
\hline Glimepiride & $109 \pm 23$ & $98 \pm 17$ & $-8.5 \pm 10.8$ & 0.003 \\
\hline \multirow[t]{2}{*}{ Mitiglinide/Voglibose } & $96 \pm 24$ & $94 \pm 21$ & $-0.8 \pm 13.0$ & 0.587 \\
\hline & 0.160 & 0.594 & 0.081 & \\
\hline \multicolumn{5}{|l|}{$\mathrm{HDL}-\mathrm{C}(\mathrm{mg} / \mathrm{dL})$} \\
\hline Glimepiride & $52 \pm 10$ & $53 \pm 13$ & $0.10 \pm 12.7$ & 0.693 \\
\hline \multirow[t]{2}{*}{ Mitiglinide/Voglibose } & $51 \pm 9.5$ & $52 \pm 9.5$ & $2.3 \pm 10.9$ & 0.612 \\
\hline & 0.842 & 0.881 & 0.725 & \\
\hline \multicolumn{5}{|l|}{ TG (mg/dL) } \\
\hline Glimepiride & $140 \pm 65$ & $132 \pm 49$ & $-3.2 \pm 31.3$ & 0.601 \\
\hline \multirow[t]{2}{*}{ Mitiglinide/Voglibose } & $102 \pm 62$ & $90 \pm 45$ & $-1.2 \pm 37.4$ & 0.192 \\
\hline & 0.114 & 0.019 & 0.725 & \\
\hline \multicolumn{5}{|l|}{ аро B (mg/dL) } \\
\hline Glimepiride & $95 \pm 18$ & $87 \pm 14$ & $-7.8 \pm 9.6$ & 0.006 \\
\hline \multirow[t]{2}{*}{ Mitiglinide/Voglibose } & $85 \pm 19$ & $75 \pm 19$ & $-6.3 \pm 10.1$ & 0.040 \\
\hline & 0.167 & 0.144 & 0.784 & \\
\hline \multicolumn{5}{|l|}{ sd-LDL (mg/dL) } \\
\hline Glimepiride & $43.5 \pm 15.3$ & $37.5 \pm 9.9$ & $-9.0 \pm 20.2$ & 0.027 \\
\hline \multirow[t]{2}{*}{ Mitiglinide/Voglibose } & $36.1 \pm 15.3$ & $32.3 \pm 12.4$ & $-8.6 \pm 17.9$ & 0.045 \\
\hline & 0.203 & 0.214 & 0.444 & \\
\hline \multicolumn{5}{|l|}{ sd-LDL proportion (\%) } \\
\hline Glimepiride & $39.9 \pm 9.9$ & $38.7 \pm 10.2$ & $-1.3 \pm 13.8$ & 0.562 \\
\hline \multirow[t]{2}{*}{ Mitiglinide/Voglibose } & $37.1 \pm 10.3$ & $33.8 \pm 9.0$ & $-7.9 \pm 11.2$ & 0.010 \\
\hline & 0.505 & 0.183 & 0.165 & \\
\hline \multicolumn{5}{|l|}{ LDL-C/apoB } \\
\hline Glimepiride & $1.15 \pm 0.10$ & $1.13 \pm 0.13$ & $-1.0 \pm 7.0$ & 0.560 \\
\hline \multirow[t]{2}{*}{ Mitiglinide/Voglibose } & $1.13 \pm 0.10$ & $1.20 \pm 0.13$ & $5.8 \pm 5.7$ & 0.002 \\
\hline & 0.704 & 0.179 & 0.007 & \\
\hline
\end{tabular}

FBG: fasting blood glucose; Hb: hemoglobin; TC: total cholesterol; LDL: low-density lipoprotein; HDL: high-density lipoprotein; TG: trigyceride; apo: apolipoprotein; sd: small-dense; sd-LDL proportion indicates the ratio of sd-LDL to LDL-C; LDL-C/apo B indicates a rough marker of LDL particle size

catabolism through insulin secretion [15]. In addition, it is reasonable that when serum LDL-C levels are reduced, levels of sd-LDL, which constitutes LDL, are also reduced. It has also been reported that glimepiride has antiatherosclerotic effects such as an LDL oxidation inhibitory effect [16-18]. However, mitiglinide/voglibose fixed-dose combination did not change serum LDL-C levels significantly, but reduced the ratio of sd-LDL to LDL, i.e., sd-LDL proportion, and increased the mean particle size of all LDL particles, which is represented by LDL-C/apoB, indicating that mitiglinide/voglibose fixed-dose combination has an effect of inhibiting LDL-induced arteriosclerosis, an effect different from that of glimepiride $[13,19]$.

It has been clearly reported that improvement in glycemic control reduces serum sd-LDL levels [20]. However, LDL-heterogeneity may be affected differently depending on the dynamics of changes in blood glucose levels. Glimepiride is a long-acting agent mainly suppressing FBG levels [4]. In contrast, mitiglinide/ voglibose fixed-dose combination is a rapid- and short-acting agent strongly suppressing postprandial blood glucose levels [10]. Cheng et al. reported that the lower the glucose AUC, i.e., the smaller the change in blood glucose levels, the lower the serum sd-LDL level. Namely, suppression of postprandial hyperglycemia by mitiglinide/voglibose fixed-dose combination may have reduced daily fluctuations in blood glucose levels and thereby improved LDL-heterogeneity [11].

The rate of reduction of FBG levels was higher in the glimepiride group than in the mitiglinide/voglibose group, while the rate of reduction of $\mathrm{HbA1c}$ levels tended to be lower in the glimepiride group, although no statistically significant differences were found in the results of our study due to the small number of patients. From the above, it is speculated that the rate of change of HbA1c levels tended to be low in the glimepiride group, because the effect of glimepiride to suppress postprandial hyperglycemia is smaller than that of mitiglinide/voglibose fixed-dose combination and thereby daily fluctuations in blood glucose levels were greater in the glimepiride group. The blood glucose AUC has been reported to be lower in a mitiglinide group than in a glimepiride group, and it seems reasonable that the concomitant administration of voglibose, which has a strong effect of suppressing postprandial hyperglycemia, would further decrease the blood glucose AUC [21,22].

Other reports including ours have also shown the effect of DPP-4 inhibitors on improving LDL-heterogeneity [19,23,24]. The results of the present study suggested that mitiglinide/voglibose fixed-dose combination, as an agent used in combination with DPP-4 inhibitors, may exert an effect of further improving LDLheterogeneity and may thereby be able to inhibit the progression of arteriosclerosis [25].

\section{Study limitations}

First, daily fluctuations in blood glucose levels should be investigated using continuous glucose monitoring to clarify fluctuations in blood glucose levels after treatment with the 2 agents. In particular, postprandial blood glucose levels, which are an indicator of fluctuations in blood glucose levels, should have been measured to assess fluctuations in blood glucose levels. Second, it is unknown whether similar results would be obtained in patients with poor glycemic control and high HbA1c levels. It has to be noted that the mean HbA1c level was near $7.0 \%$ in the patients examined in this study. Monnier et al. reported that when the HbA1c level is $\leq 7.3$, postprandial blood glucose levels more strongly influence glycemic control than preprandial blood glucose levels [26], and it cannot be ruled out that effects of the 2 agents on LDL-heterogeneity may be different depending on the HbA1c level. Third, it is also necessary to consider fluctuations in TG levels, particularly postprandial TG levels, which greatly affect LDL-heterogeneity [27]. Last, we cannot draw a conclusion about which agent had the greater antiatherosclerotic effect using this study design. We expect that this pilot study will be the basis for large-scale clinical studies.

\section{Conclusion}

The administration of mitiglinide/voglibose fixed-dose combination in addition to DPP-4 inhibitors improved glycemic con- 


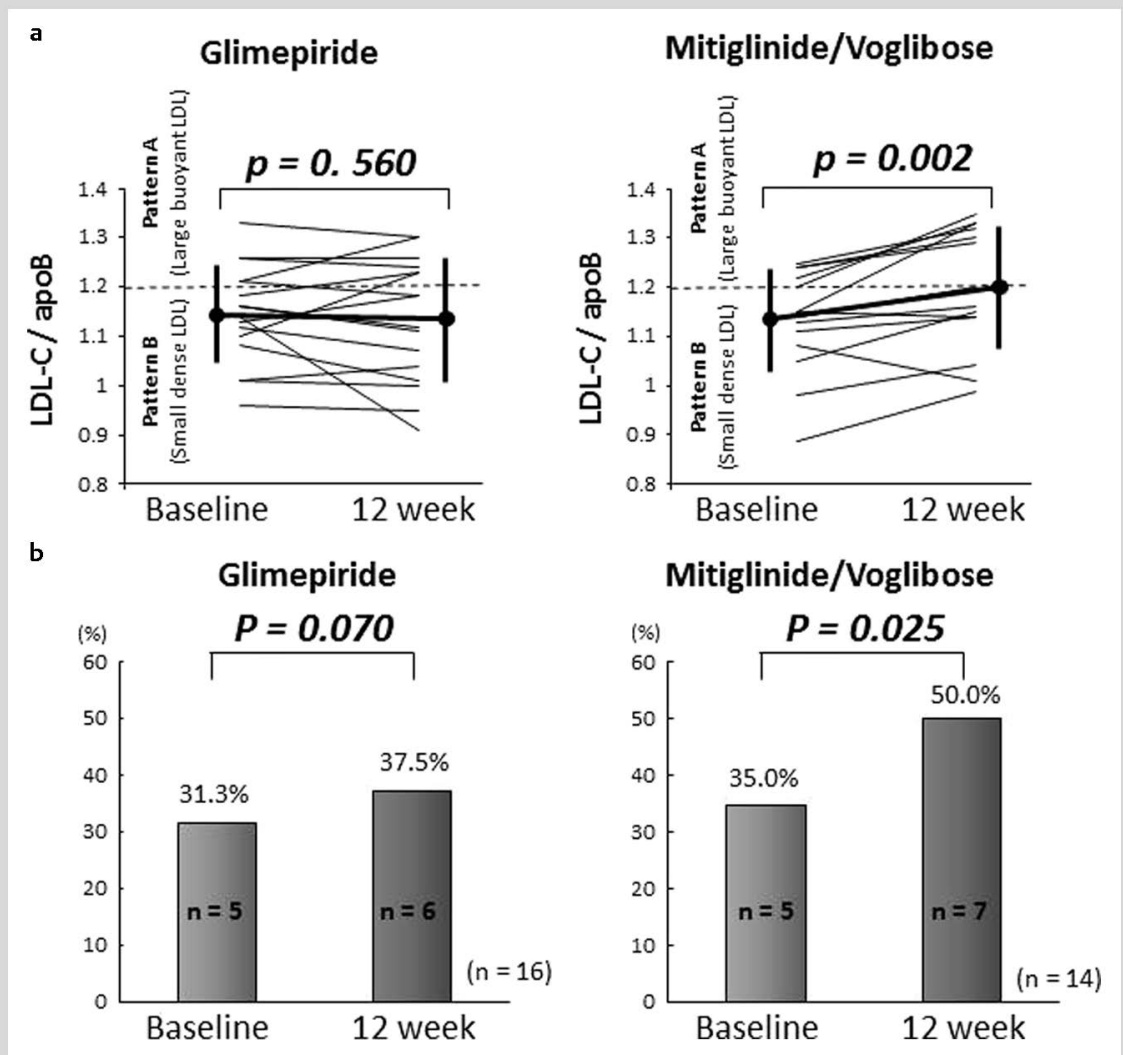

Fig. 2 Changes in LDL-heterogeneity. LDL-C: low-density lipoprotein cholesterol, apo: apolipoprotein. a Changes in mean ( \pm SD) LDL-C/ apoB as estimated LDL particle size at week 12 vs. baseline. b Percentage attaining LDL-C/apoB Target ( $\geq 1.2)$ LDL-C/apo B ratio of 1.2 corresponded to a LDL diameter of $25.5 \mathrm{~nm}$, which is the cut-off value to distinguishing LDL pattern A (large buoyant LDL) from $B$ (small dense LDL) [13]. trol to a degree comparable to that of glimepiride, but the results suggested that this combination affects LD- metabolism differently compared with glimepiride. This may be related to fluctuations in blood glucose levels after treatment with these agents.

\section{Declaration of Interest}

$\nabla$

All the authors declare that they have no competing interests.

\section{References}

1 Ohkubo Y, Kishikawa H, Araki E et al. Intensive insulin therapy prevents the progression of diabetic microvascular complications in Japanese patients with non-insulin-dependent diabetes mellitus: a randomized prospective 6-year study. Diabetes Res Clin Pract 1995; 28: 103-117

2 Nathan DM, Buse JB, Davidson MB et al., American Diabetes Association; European Association for Study of Diabetes. Medical management of hyperglycemia in type 2 diabetes: a consensus algorithm for the initiation and adjustment of therapy: a consensus statement of the American Diabetes Association and the European Association for the Study of Diabetes. Diabetes Care 2009; 32: 193-203

3 Ono Y, Kamoshima H, Nakamura A et al. Glycemic/metabolic responses to identical meal tolerance tests at breakfast, lunch and dinner in Japanese patients with type 2 diabetes mellitus treated with a dipeptidyl peptidase-4inhibitor and the effects of adding a mitiglinide/ voglibose fixed-dose combination. Expert Opin Pharmacother 2014; 15: 1785-1795

4 Wu L, Parhofer KG. Diabetic dyslipidemia. Metabolism 2014; 63: 1469-1479

5 Rana JS, Dunning A, Achenbach $S$ et al. Differences in prevalence, extent, severity, and prognosis of coronary artery disease among patients with and without diabetes undergoing coronary computed tomography angiography: results from 10,110 individuals from the CONFIRM (COronary CT Angiography EvaluatioN For Clinical Outcomes): an InteRnational Multicenter Registry. Diabetes Care 2012; 35: 1787-1794

6 Geisen K. Special pharmacology of the new sulfonylurea glimepiride. Arzneimittelforschung 1988; 38: 1120-1130
7 Xu DY, Zhao SP, Huang QX et al. Effects of Glimepiride on metabolic parameters and cardiovascular risk factors in patients with newly diagnosed type 2 diabetes mellitus. Diabetes Res Clin Pract 2010; 88: 71-75

8 Umpierrez G, Issa M, Vlajnic A. Glimepiride versus pioglitazone combination therapy in subjects with type 2 diabetes inadequately controlled on metformin monotherapy: results of a randomized clinical trial. Curr Med Res Opin 2006; 22: 751-759

9 Derosa G, Gaddi AV, Piccinni MN et al. Differential effect of glimepiride and rosiglitazone on metabolic control of type 2 diabetic patients treated with metformin: a randomized, double-blind, clinical trial. Diabetes Obes Metab 2006; 8: 197-205

10 Langenfeld MR, Forst T, Hohberg C et al. Pioglitazone decreases carotid intima-media thickness independently of glycemic control in patients with type 2 diabetes mellitus: results from a controlled randomized study. Circulation 2005; 11: 2525-2531

11 Inoue $M$. Tighter control of postprandial hyperglycemia with mitiglinide/voglibose fixed-dose combination in Japanese patients with type 2 diabetes mellitus. Expert Opin Pharmacother 2012; 13: 2257-2268

12 Ding $\mathrm{C}, \mathrm{Hsu} \mathrm{SH}, \mathrm{Wu} \mathrm{YJ}$ et al. Additive effects of postchallenge hyperglycemia and low-density lipoprotein particles on the risk of arterial stiffness in healthy adults. Lipids Health Dis 2014; 13: 179

13 Hirano T, Ito $Y$, Yoshino G. Measurement of small dense low-density lipoprotein particles. J Atheroscler Thromb 2005; 12: 67-72

14 Sniderman $A D$, Blank $D$, Zakarian $R$ et al. Triglycerides and small dense LDL: the twin Achilles heels of the Friedewald formula. Clin Biochem 2003; 36: 499-504

15 Schaalan M, El-Abhar HS, Barakat M et al. Westernized-like-diet-fed rats: effect on glucose homeostasis, lipid profile, and adipocyte hormones and their modulation by rosiglitazone and glimepiride. J Diabetes Complications 2009; 23: 199-208

16 Shakuto S, Oshima K, Tsuchiya E. Glimepiride exhibits prophylactic effect on atherosclerosis in cholesterol-fed rabbits. Atherosclerosis 2005; 182: 209-217

17 Hadi NR, Al-Amran F, Hussein MA et al. Evaluation of the effects of glimepiride (Amaryl) and repaglinide (novoNorm) on atherosclerosis progression in high cholesterol-fed male rabbits. J Cardiovasc Dis Res 2012; 3: 5-11

18 Katakami $N$, Kaneto $H$, Matsuhisa $M$ et al. Effects of glimepiride and glibenclamide on carotid atherosclerosis in type 2 diabetic patients. Diabetes Res Clin Pract 2011; 92: e20-e22 
19 Tani S, Nagao K, Hirayama A. Association between urinary albumin excretion and low-density lipoprotein heterogeneity following treatment of type 2 diabetes patients with the dipeptidyl peptidase- 4 inhibitor, vildagliptin: a pilot study. Am J Cardiovasc Drugs 2013; 13: 443-450

20 Hirano T, Naito H, Kurokawa $M$ et al. High prevalence of small LDL particles in non-insulin-dependent diabetic patients with nephropathy. Atherosclerosis 1996; 123: 57-72

21 Ohkura T, Inoue K, Fujioka $Y$ et al. The proinsulin/insulin (PI/I) ratio is reduced by postprandial targeting therapy in type 2 diabetes mellitus: a small-scale clinical study. BMC Res Notes 2013; 6: 453

22 Konya $H$, Katsuno $T$, Tsunoda $T$ et al. Effects of combination therapy with mitiglinide and voglibose on postprandial plasma glucose in patients with type 2 diabetes mellitus. Diabetes Metab Syndr Obes 2013; 6: 317-325

23 Terasaki M, Nagashima M, Watanabe T et al. Effects of PKF275-055, a dipeptidyl peptidase- 4 inhibitor, on the development of atherosclerotic lesions in apolipoprotein E-null mice. Metabolism 2012; 61: 974-977
24 Terasaki M, Nagashima M, Nohtomi K et al. Preventive effect of dipeptidyl peptidase-4 inhibitor on atherosclerosis is mainly attributable to incretin's actions in nondiabetic and diabetic apolipoprotein E-null mice. PLoS One 2013; 8: e70933

25 Kwiterovich PO Jr. Clinical relevance of the biochemical, metabolic, and genetic factors that influence low-density lipoprotein heterogeneity. Am J Cardiol 2002; 90: 30i-47i

26 Monnier L, Lapinski H, Colette C. Contributions of fasting and postprandial plasma glucose increments to the overall diurnal hyperglycemia of type 2 diabetic patients: variations with increasing levels of $\mathrm{HbA}(1 \mathrm{c})$. Diabetes Care 2003; 26: 881-588

27 Tani S, Matsumoto M, Nagao $\mathrm{K}$ et al. Association of triglyceride-rich lipoproteins-related markers and low-density lipoprotein heterogeneity with cardiovascular risk: effectiveness of polyacrylamide-gel electrophoresis as a method of determining low-density lipoprotein particle size. J Cardiol 2014; 63: 60-68 